\title{
A SEARCH FOR INFRARED POLARIZATION \\ IN THE GALACTIC CENTER
}

T. MAIHARA, H. OKUDA, and S. SATO

Dept. of Physics, Kyoto University, Kyoto, Japan

\begin{abstract}
Observations of infrared polarization have been tried in the galactic center. A preliminary result is that the polarization at $\mathrm{K}$-band $(2.2 \mu)$ is less than $5 \%$, much less than that expected from interstellar polarization.
\end{abstract}

\section{Introduction}

A few years ago, Becklin and Neugebauer discovered a strong near infrared source coinciding with the position of the galactic center (Becklin and Neligebauer, 1968). Extension to longer wavelength observations by themselves and by Low and Rieke have revealed some strong middle infrared radiation in more compact regions (Becklin and Neugebauer, 1969; Rieke and Low, 1971). Furthermore, surprisingly strong emission has also been found in the far infrared region by Low's observations from a jet-plane and by Hoffmann and others using balloon borne equipment. (Aumann and Low, 1970; Hoffmann et al., 1971). The emission mechanisms of these radiations have not been well understood.

Measurement of polarization in the galactic center could give some information regarding these emission mechanisms. Low and others have observed it in middle infrared and obtained an upper limit of about $3 \%$ for the polarization at $10 \mu$ (Low et al., 1969).

Strong infrared emission of the galactic center may also be useful for the investigation of interstellar space especially in the central part of the Galaxy, which is inaccessible by ordinary methods.

Extremely large interstellar extinction of the galactic center would be accompanied by large polarization, provided the interstellar dust particles are partially aligned by a galactic magnetic field or some other mechanism. This is another reason why we tried to measure the infrared polarization in the galactic center.

Since the preliminary report at the Liège symposium (Maihara et al., 1972), we have made observations in 1971 and 1972 by a somewhat different method from the previous one.

\section{Observations}

The infrared polarimeter which we used is the same as used in the measurements of polarization of the infrared stars (Hashimoto et al., 1970). The details of the polarimeter will be published elsewhere (Maihara et al., 1973). An HR type polaroid put 
between the filter and the detector was rotated with a constant speed of one rotation in about $2 \mathrm{~min}$.

The observations were carried out in the summers of 1970, 1971 and 1972 by attaching the polarimeter to the 36 or 74-in. reflector at Okayama Astrophysical Observatory. The diaphragm was $5 \mathrm{~mm}$ in diameter so that fields of $30^{\prime \prime}$ (74-in.) or 1' (36-in.) were available. The observations were done in so-called $\mathrm{K}$-band or at 2.2. $\mu$ by using a liquid nitrogen cooled lead sulphide photoconductor.

Since the signal from the galactic center is so weak observations of many rotations were necessary to improve the statistics. The signal from the detector was recorded on a magnetic tape together with clock pulses which indicate the rotation angle of the polarizer. In the observations of 1970 , the data was processed by an analog method, that is, the signals for each rotation of the polarizer were added to preceding data after rewinding the tape and reading it out. From the observations of 1971, the signals were recorded in series for a number of rotations to save the dead time for rewinding the tape. After the observations, they were digitised with a constant sample rate, added and analysed by a computer.

The results thus analysed are given in Table I, which are also illustrated in Figure I. The position angle is measured eastward from the meridian.

Finite degrees of polarization seem to be present statistically. But we must be careful to check for spurious polarizations of instrumental origin. In order to check such instrumental polarizations, we examined the following points.

First, the Fourier components of the first and the fourth harmonics were calculated as well as the second harmonics. Only the second harmonics-which are responsible for the polarization-appear to be significantly larger than the statistical fluctuations.

Second, the polarizations of normal stars were measured. They are much less than the observed values for the galactic center.

TABLE I

Infrared polarization of the galactic center

\begin{tabular}{lllc}
\hline Date & Rotations & Amplitude & Position angle \\
\hline 1970 Jul. & 57 & $5.3 \pm 1.3 \%$ & $19^{\circ} \pm 7.5^{\circ}$ \\
1971 May & 26 & $7.4 \pm 2.7$ & $-22.6 \pm 15$ \\
1972 May & 34 & $6.0 \pm 3.5$ & $-31 \pm 20$ \\
& &
\end{tabular}

\begin{tabular}{llll}
\hline & 1st & 2nd & 4th \\
\hline 1971 May & $2.3 \pm 2.3 \%$ & $7.4 \pm 2.7 \%$ & $4.3 \pm 2.3 \%$ \\
1972 May & $3.9 \pm 3.3$ & $6.0 \pm 3.5$ & $4.2 \pm 3.3$
\end{tabular}

Calibration by stars

$\begin{array}{ll}\alpha \text { Sco } & 1.4 \pm 0.2 \% \\ \gamma \text { Leo } & 0.6 \pm 0.2\end{array}$

Polarization of sky background

34 rotations $\quad 1.8 \pm 1.9$ 


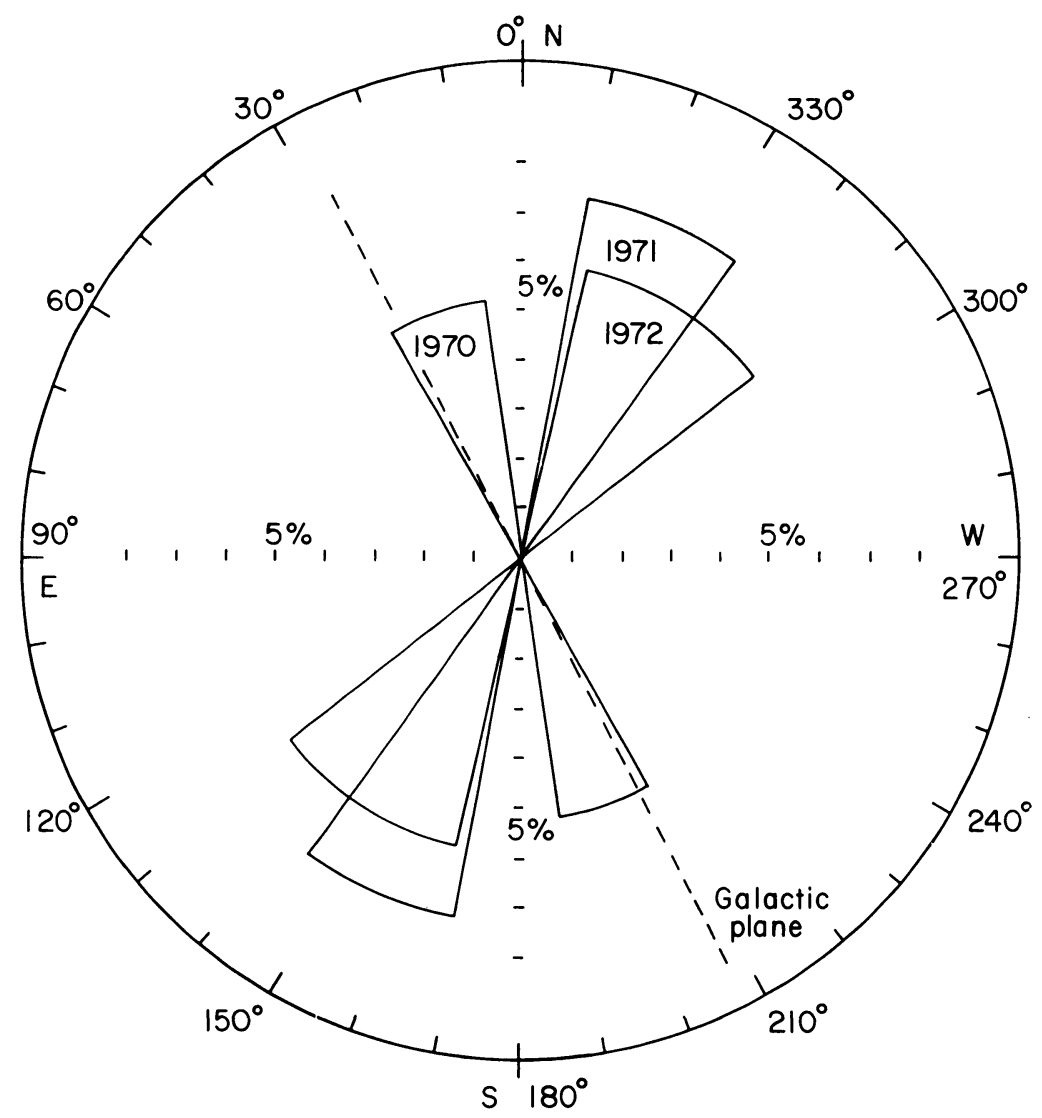

Fig. 1. Infrared polarizations toward the galactic center observed in 1970, 1971 and 1972.

Third, the signal from the sky background was checked. It did not show any significant polarization larger than the statistical fluctuation.

Results of these checks are also given in Table I.

\section{Discussion}

So far as we have checked the data, no spurious polarization was definitely seen. But we must note that the observational conditions were rather poor, that is, only a short time was available and elevation of the galactic center was low in the southern sky so that signal to noise ratio was very poor. We therefore reserve our final conclusion to the more detailed observations in the future and take the observed values of about $5 \%$ as an upper limit. Even in this case, the following discussion may be interesting.

That polarization may appear in the course of propagation through interstellar space has been well known in the optical region. Mathewson and Ford have made intensive studies of interstellar polarization of stars (Mathewson and Ford, 1970). From their data we obtained the degree of polarization and position angles of these 


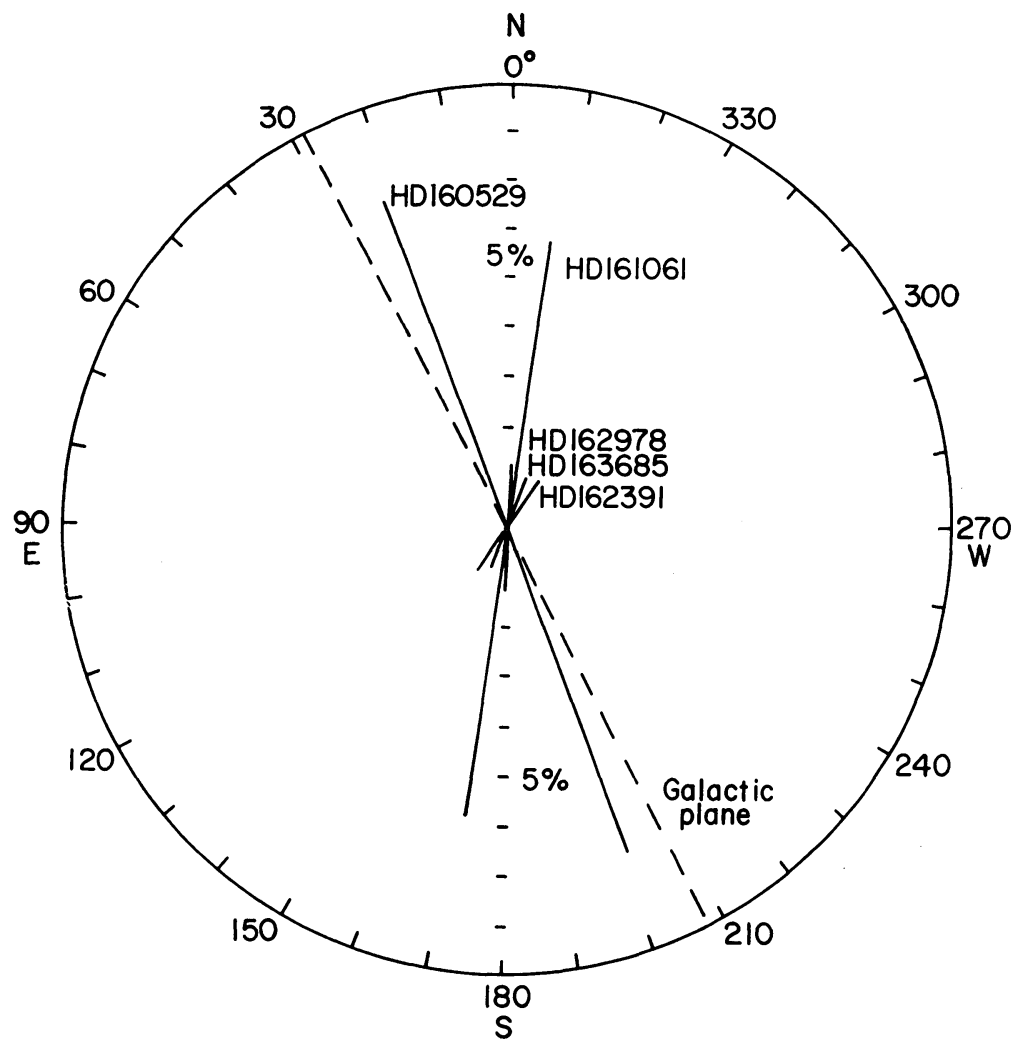

Fig. 2. Insterstellar polarization of stars near the galactic center.

stars which are located within $5^{\circ}$ from the galactic center. These are shown in Figure 2. HD 161061 is the nearest star to the galactic center (about $1^{\circ}$ separation). The degree of polarization of these stars is relatively large and the position angles are concentrated in a narrow range of direction, almost coincident with our observations shown in Figure 1.

Interstellar polarization is correlated with extinction. In Figure 3, the relation between the degree of polarization and the amount of visual extinction is shown by plotting the data of Mathewson and Ford (Mathewson and Ford, 1970). The stars near the galactic center are marked by dots with a circle. It is seen that the degree of polarization generally is proportional to the extinction. On the other hand, interstellar polarization decreases with wavelength; about one third of visual polarization would be expected for K-band (Dyck, 1972).

If we adopt $27 \mathrm{mag}$. of visual extinction for the galactic center as was estimated by Becklin and Neugebauer (1968) and assume that the above relations of interstellar polarization hold in the whole path to the galactic center, the polarization at K-band would be expected as large as 10 to $20 \%$. This is much larger than the observed upper limit. 


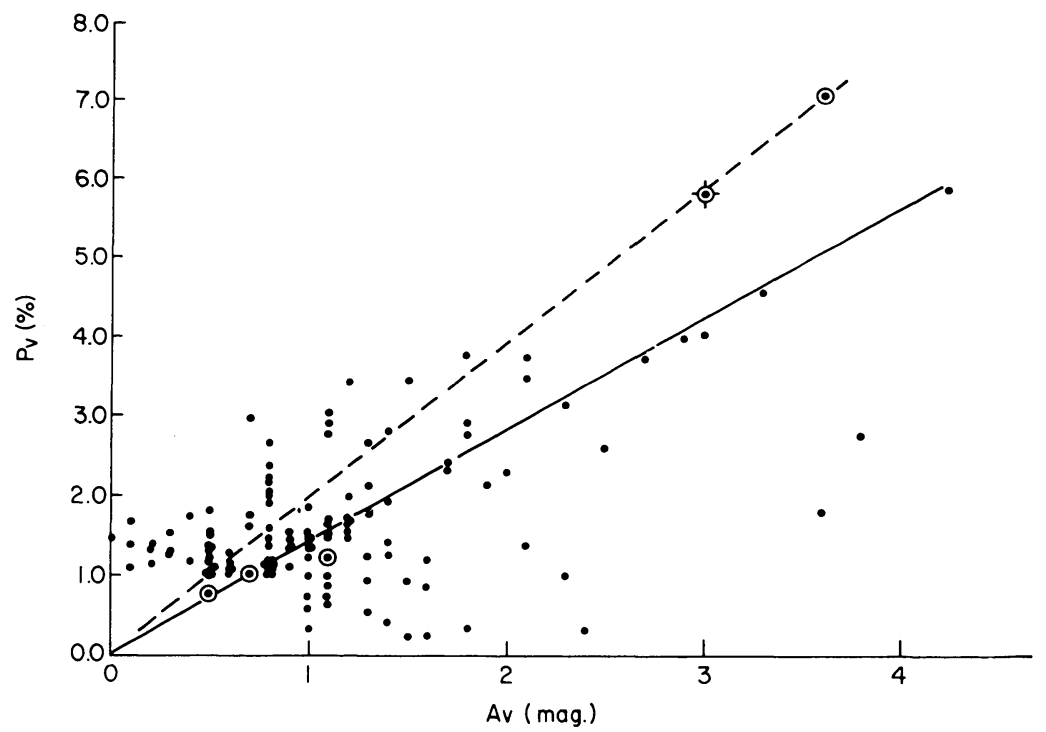

Fig. 3. Relation between the degree of interstellar polarization and the visual extinction. Stars near the galactic center are indicated by dots with a circle.

As the interstellar polarization is believed to be caused by non spherical dust grains aligned by a regular magnetic field in the Galaxy, the observations might suggest that the magnetic field in the inner part of the Galaxy is not so regular as seen in the solar neiborhood. Stephen Loyd and Harwit have obtained a similar result by their detailed studies of the differential polarization of starlight as a function of distance (Stephen Loyd and Harwit, 1973).

The polarization of the galactic center might also be due in part to scattering of light from the central source by dust grains which are distributed asymmetrically or disk-like around the galactic center, as has been found in infrared stars (Hashimoto et al., 1970; Forbes, 1971; Dyck et al., 1917).

In any case, we must limit further dicussions until more detailed and definite observations are made. We are now preparing to make another observation next year and hope to get more definite results.

\section{Acknowledgement}

We want to thank the staff in Okayama Astrophysical Observatory for their kind help in our observations.

\section{References}

Aumann, W. F. and Low, F. J.: 1970, Astrophys. J. 159, L159.

Becklin, E. E. and Neugebauer, G.: 1968, Astrophys. J. 151, 145.

Becklin, E. E. and Neugebauer, G.: 1969, Astrophys. J. 157, L31. 
Dyck, H. M., Forbes, F. F. and Shawl, S. J.: 1971, Astron. J. 79, 901.

Dyck, H. M.: 1972, private communication.

Forbes, F. F.: 1971, Astrophys. J. 165, L83.

Hashimoto, J., Maihara, T., Okuda, H., and Sato, S. : 1970, Publ. Astron. Soc. Japan 22, 335.

Hoffmann, W. F., Frederick, C. L., and Emery, R. J.: 1971, Astrophys. J. 164, L23.

Lloyd, S. and Harwit, M.: 1973, this volume, p. 203.

Low, F. J., Kleinmann, D. E., Forbes, F. F., and Aumann, H. H.: 1969, Astrophys. J. 157 , L97.

Maihara, T., Okuda, H., and Sato, S.: 1972, Mém. Soc. Roy. Sci. Liège, 6e sér. Tome III, p. 417.

Maihara, T., Okuda, H., and Sato, S.: 1973, Memoir of the Faculty of Science, Kyoto University, in print.

Mathewson, D. S. and Ford, V. L.: 1970, Mem. Roy. Astron. Soc. 74, 139.

Rieke, G. H. and Low, F. J.: 1971, Nature 233, 53. 